\title{
Continuità e mutamento nella teoria dell'interpretazione di Umberto Eco
}

Quando nel 1962 vennero raccolti e pubblicati i saggi che costituiscono Opera aperta, tra le varie voci del dibattito suscitato da quel libro innovativo non mancarono quelle di chi accusò il giovane Umberto Eco di legittimare con le proprie tesi una sorta di anarchismo interpretativo, che produceva l'esito di rendere, di fatto, inutile l'attività critica, autorizzando ciascuno a dire qualcosa di diverso dall'altro. 'A distanza di circa trent'anni da quel fortunato e controverso precedente, e dopo i successi, oltre che dei suoi libri teorici, anche delle sue opere narrative, Umberto Eco ha pubblicato un libro dichiaratamente polemico nei confronti dei fautori di una incondizionata libertà ermeneutica, dedicandolo proprio alla definizione dei limiti dell'interpretazione. Una scelta, questa, che ha fatto molto discutere, e che ha indotto alcuni a pensare ad un voltafaccia di quello che era sempre stato considerato come uno dei più strenui difensori della libertà dell'interprete. Ma si è trattato davvero solo della conversione di uno scrittore affermato, che, avendo ormai raggiunto il successo, ha finito per arroccarsi su posizioni "conservatrici"? O si è trattato, piuttosto, della lucida strategia di un autore che snobisticamente ha scelto, in un momento in cui tutti inneggiavano ai diritti e all'autonomia del lettore, di rivalutare la questione del rigore dell'interpretazione, cos̀ come anni fa aveva scelto di enfatizzare l'apertura dell'opera ad un ventaglio infinito di interpretazioni, quando la critica era in larga misura attestata su posizioni obiettivistiche?

$\mathrm{Nel}$ suo libro Eco previene l'accusa di aver dato una svolta conservatrice al proprio pensiero e rivendica la coerenza della sua riflessione intorno al tema dell'interpretazione: "Trent'anni fa—scrive nell'introduzione al volume-partendo anche dalla teoria dell'interpretazione di Luigi Pareyson, mi preoccupavo di definire una sorta di oscillazione, o di instabile equilibrio, tra iniziativa dell'interprete e fedeltà all'opera. Nel corso di questi trent'anni qualcuno si è sbilanciato troppo sul versante dell'iniziativa dell'interprete. Il problema ora non è di sbilanciarsi in senso opposto, bensì di sottolineare ancora una volta l'ineliminabilità dell'oscillazione" (Limiti 13-14). Sarebbe quindi innanzitutto la preoccupazione delle estremizzazioni a cui sono state portate alcune istanze del dibattito culturale recente ad averlo spinto a questa messa a punto teorica, che è dunque possibile leggere anche e soprattutto come un segno dei mutamenti intervenuti nel panorama culturale degli ultimi trent'anni.

All'epoca di Opera aperta, Eco si proponeva l'ambizioso traguardo di inda- 
gare la reazione della sensibilità contemporanea "in risposta alle suggestioni della matematica, della biologia, della fisica, della psicologia, della logica e del nuovo orizzonte epistemologico che queste scienze hanno aperto" (Opera 2). Questo nella convinzione che tale reazione si esprimesse attraverso le opere d'arte, le quali da sempre, in quanto prodotto di una data sensibilità, rappresentano un'immagine del mondo che ha il valore di una metafora epistemologica. Metafora che si rivela innanzitutto nel modo in cui un'opera si organizza, manifestando, in quanto forma, le tendenze storiche e personali che hanno presieduto al suo concretarsi e quindi l'implicita visione del mondo che un certo modo di formare presuppone. Il confronto tra le modalità d'organizzazione dell'opera e la realtà di altri ambiti del sapere gli sembrava poter avvenire sulla base di un concetto di struttura, intesa come sistema di relazioni al quale l'opera può essere analiticamente ricondotta. Se, quindi, caratteristica dell'arte in generale è quella di essere un messaggio fondamentalmente ambiguo, nel quale convivono una pluralità di significati, Eco notava come l'organizzazione strutturale di molte opere contemporanee, come le composizioni musicali di Berio e Stockhausen, traducesse questa condizione in una esplicita finalità artistica, rifacendosi a quegli ideali di informalità, disordine ed indeterminazjone che contraddistinguono l'orizzonte conoscitivo dell'uomo contemporaneo. Peculiarità di queste opere era quelia di presentarsi incompiute al fruitore, che veniva chiamato a portarle a compimento, trovandosi così posto al centro di una rete di relazioni virtuali, tra le quali egli instaurava la propria forma senza essere determinato da una necessità. Tale disponibilità, o apertura, dell'opera si distingueva, allora, da quella tipica di ogni opera d'arte in sé compiuta, che-come aveva mostrato Luigi Pareysonnell'incontro con il proprio fruitore viene di volta in volta attualizzata secondo la prospettiva individuale, determinata dalla situazione esistenziale, dalla sensibilità e dalla cultura di cui l'interprete è portatore. Ciò nonostante l'opera aperta contemporanea non poteva essere intesa, secondo Eco, come un invito all'indiscriminato, ma piuttosto come un campo di possibilità, che orienta e delimita le scelte del fruitore, il quale non può in ogni caso abbandonarsi ad un arbitrio assoluto, ma è guidato nelle sue decisioni dalla struttura dell'opera. La relazione fruitiva tra l'opera e l'interprete gli sembrava, quindi, caratterizzata da una dialettica tra forma e apertura, cioè dalla capacità di un'opera di realizzare la massima ambiguità e dipendere dall'intervento attivo del fruitore, senza cessare d'essere un oggetto dotato di proprietà strutturali definite, che permette e allo stesso tempo coordina il libero gioco delle interpretazioni. La tendenza al disordine che caratterizza positivamente la poetica dell'apertura, veniva dunque presentata da Eco come una "tendenza al disordine dominato, alla possibilità compresa in un campo, alla libertà sorvegliata da germi di formatività presenti nella forma che si offre aperta alle libere scelte del fruitore" (Opera 123-24).

Muovendo da queste posizioni l'itinerario teorico di Eco, in merito alla questione dell'interpretazione dell'opera d'arte, sembra essersi in seguito sviluppato lungo due principali direttrici. Da una parte-secondo una prospettiva che, sche- 
matizzando, dall'approccio presemiotico di Opera aperta arriva sino al Trattato di semiotica generale, passando per il saggio dedicato a "Le Strutture narrative in Fleming" e per La struttura assente-in aperta opposizione alle estetiche intuitive, Eco si propone di smontare analiticamente il preciso meccanismo mediante il quale l'opera realizza quella peculiare ambiguità ed autoriflessività che caratterizza il messaggio estetico, secondo la nota definizione della funzione poetica del linguaggio data da Jakobson. Sebbene l'unità dell'opera sembri manifestare una sorta di resistenza a questa dissezione analitica di tipo semiotico, secondo Eco ciò avviene proprio per lo scatenarsi di meccanismi semiotici, i quali concorrono alla costituzione del codice particolare di ogni opera, cioè alla formazione del suo idioletto. Compito dell'analisi semiologica è, allora, costruire un modello ${ }^{2}$ che rappresenti la regola generale che si pronuncia ai diversi livelli dell'opera e, allo stesso tempo, comprenda una serie di sottoregole che presiedono a quelle variazioni alla norma, che lo stesso artista introduce nel sistema di convenzioni principale. L'opera viene quindi intesa come una "super-funzione segnica" (Trattato 339), che correla diversi piani espressivi organizzandoli ambiguamente, secondo un disegno che è però identificabile. ${ }^{3}$

Secondo tale prospettiva, l'analisi semiologica può fare presa solo sull'opera in quanto messaggio-fonte, cioè in quanto codice che rappresenta il punto di partenza per una serie di libere scelte interpretative (Struttura 70), mentre l'esperienza fruitiva, che si attua durante la comunicazione estetica, è considerata irriducibile ad una misura quantitativa o ad una sistemazione strutturale. Le premesse elaborate in Opera aperta e la vocazione panottica del progetto semiotico proposto nel Trattato di semiotica generale, spingono però Eco ad avventurarsi lungo l'altra direttrice problematica verso la quale si indirizza la sua riflessione, e cioè l'indagine delle dinamiche della cooperazione interpretativa da parte del fruitore dell'opera d'arte. Convinto che nell'esaminare le possibilità significative di una struttura comunicativa non sia possibile prescindere dal polo ricettore, Eco mette in luce, in Lector in fabula, come l'epifania del contenuto semantico di un testo non possa essere considerata come una parte esplicita della sua manifestazione linguistica, così come pretendeva lo strutturalismo più intransigente, ma sia il frutto di complesse operazioni di inferenza testuale basate sulla competenza intertestuale del fruitore.

Come è quindi evidente dall'itinerario teorico che si è qui brevemente ricostruito, la questione dell'interpretazione svolge un ruolo centrale in tutta l'opera di Umberto Eco (romanzi compresi). Centralità che viene ribadita anche nel suo più recente libro teorico, nel quale l'interpretazione è intesa come un fenomeno che si estende al di là dell'ambito comunicativo in senso stretto e coinvolge gli stessi processi percettivi, rappresentando il "meccanismo semiosico che spiega non solo il nostro rapporto con messaggi elaborati intenzionalmente da altri esseri umani, ma ogni forma di interazione dell'uomo (e forse degli animali) con il mondo circostante" (Limiti 12).

Negli ultimi decenni un sensibile mutamento di paradigma è intervenuto nel- 
l'orizzonte degli studi letterari, nel cui ambito si è assistito ad un proliferare di nuovi indirizzi metodologici e ad un progressivo spostamento del dibattito dall'immanentismo testuale dell'atteggiamento strutturalista verso una pragmatica della lettura. Tale trasformazione nasce come una reazione all'irrigidimento obiettivistico di alcune frange dello strutturalismo, così come, in area anglosassone, ai limiti manifesti del dominante New Criticism, che pretendeva di isolare il testo poetico da ogni contestualità, considerandolo nella sua autonomia semantica assoluta di "icona verbale" (Wimsatt Jr. e Beardsley). Secondo Eco, la tendenza a privilegiare, nell'analisi critica, il momento della fruizione affonda le proprie radici storiche in una tradizione estetica a orientamento interpretativo- a cui appartengono la concezione aristotelica della catarsi, quella pseudolonginiana del sublime, le estetiche medievali della visione, fino ad arrivare al sublime kantiano e a numerose estetiche contemporanee-e si manifesta quindi come rivalutazione di una tradizione precedente che sino ad ora sarebbe stata lasciata in penombra. Tale evoluzione del dibattito critico recente può d'altronde anche essere letta nel segno della continuità con istanze portate in luce dallo stesso strutturalismo e dalla semiotica, la cui ricerca di modelli descrittivi delle strutture e dei codici sottesi alla produzione del senso ha portato ad una concezione dell'opera come costrutto intertestuale, prodotto di diversi discorsi culturali che si polarizzano appunto nel momento della lettura.

Aspetto singolare del nuovo panorama critico è il suo frammentarsi in una pluralità di indirizzi differenti, il cui comune denominatore è l'idea che l'opera debba essere avvicinata, non tanto indagando il suo momento generativo, quanto interrogandosi sul ruolo che viene svolto dal destinatario nella sua comprensione ed interpretazione, nonché sulle modalità in cui il testo prevede questa partecipazione. Sebbene ancora non molto evidente in Italia, dove, grazie ad un atteggiamento particolarmente flessibile, il modello strutturalistico-semiologico è riuscito a mantenere più a lungo la propria egemonia (Di Girolamo et al. 3-7), questa polverizzazione delle componenti del dibattito critico-metodologico ha forse la sua massima espressione in America. ${ }^{4}$ Ed è proprio in America che si è affermata negli studi letterari quella critica decostruzionista, che rappresenta il principale bersaglio verso cui si indirizzano gli attacchi polemici espressi da Eco ne $I$ limiti dell' interpretazione.

L'avvento del decostruzionismo letterario in America viene di solito fatto risalire al 1966, ed in particolare alla conferenza pronunciata da Jacques Derrida ${ }^{5}$ nell'ambito del convegno intitolato The Language of Criticism and the Sciences of Man, organizzato dal John Hopkins Humanities Center. In questa occasione Derrida parla dell'esaurimento e delle aporie del pensiero strutturalista, affermando una prospettiva in cui l'interpretazione non è più l'oggettivo accertamento da parte del lettore dei significati intesi dall'autore, ma libero gioco ermeneutico, interpretazione infinita secondo le nozioni di differenza e disseminazione. Questa posizione si traduce in una pratica volta a "decostruire" il testo, minandone l'apparente solidità interna, mettendone in evidenza le "tracce", i nessi, le 
citazioni e le interferenze. A partire da queste suggestioni si sono sviluppate in America tutta una serie di correnti critiche ispirate alla riflessione derridiana, tra le quali quella forse più nota è quella dei cosiddetti "Yale Critics" (Paul De Man, Geoffrey Hartman, J. Hillis Miller, Harold Bloom).

Molti degli interventi di Eco intorno alla questione dei limiti dell'interpretazione nascono con un intento apertamente polemico nei confronti del vistoso spostamento d'accento sull'iniziativa del destinatario proposto dal decostruzionismo. Già in La struttura assente, Eco aveva sottolineato come il tentativo di dissoluzione del pensiero strutturale attuato dalla filosofia di Derrida e di Michel Foucault portasse al paralizzante approdo di un'ontologia dell'Assenza, "tale da piegarci a un riconoscimento silenzioso della Necessita, e da bloccare ogni progetto di contestazione delle cose così come sono" (9), e ad esso aveva opposto la proposta di un recupero operativo del concetto di struttura come modello conoscitivo, come strumento ipotetico per muoversi nell'universo dei rapporti storici e sociali. Al dilagante decostruzionismo letterario, che alla filosofia di Derrida esplicitamente si richiama, Eco rimprovera ora di relegare di fatto il testo alla funzione di mero stimolo per una deriva interpretativa, che non produce altro risultato se non quello di fornire alla scrittura dei pretesti per riprodursi. Ed è proprio quest'idea dello slittamento perpetuo del senso ad accomunare, secondo Eco, molte teorie postmoderne della critica a quell'atteggiamento intellettuale che chiama "semiosi ermetica", 6 e che fa risalire al diffondersi dell'ermetismo e dello gnosticismo in ambiente ellenistico, a partire dal II secolo d.C. Caratteristica del pensiero ermetico è la ricerca di una verità sconosciuta ed inattingibile, di cui però ogni libro parla per mezzo di misteriose allusioni ed allegorie, secondo una concezione che trasforma l'intero teatro del mondo in un fitto intrecciarsi di corrispondenze significative, per cui ogni oggetto o evento è ridotto a fenomeno linguistico, ma è contemporaneamente privato di ogni potere comunicativo, in quanto trasmette un significato ineffabile, raggiungibile solo per via intuitiva. Tali caratteristiche dell'ermetismo classico sopravvivono poi nel medioevo e si diffondono in epoca rinascimentale, tanto da influenzare addirittura la nascita di quella scienza moderna, che diverrà di lì a poco uno dei più accaniti avversari dell'atteggiamento ermetico, il quale continua però a sopravvivere diffondendosi tra $\mathrm{i}$ poeti ed i filosofi, da Goethe a Nerval a Yeats, da Heidegger a Jung. Ad accomunare semiosi ermetica e deriva decostruzionista è una particolare degenerazione del processo semiotico di produzione del significato, che porta ad una sorta di sviluppo canceroso delle connotazioni, per cui ad un certo punto della catena degli interpretanti di un segno un'associazione semplicemente fonetica "apre una nuova catena pseudo-connotativa in cui il Contenuto del nuovo segno non dipende più dal Contenuto del primo" (Limiti 328). Così a mano a mano che si procede nella catena delle corrispondenze si perde ogni traccia del segno di partenza, e ci si abbandona al piacere del vagabondaggio in un universo trasformato in foresta di simboli.

Eco è poi particolarmente critico verso le teorie che sostengono l'idea che 
ogni interpretazione non sia che una misinterpretazione (neologismo che traduce il termine inglese misreading). Secondo tali posizioni, interpretare un testo significa sempre e comunque in parte fraintenderlo, e cioè tradime gli intenti significativi, arricchendolo o limitandolo mediante una lettura condizionata storicamente ed esistenzialmente. Da tale consapevolezza alcuni critici sono partiti per legittimare l'idea che tutte le interpretazioni si equivalgano e che sia sostanzialmente impredicabile qualsiasi criterio di pertinenza per stabilire la validità della lettura di un dato testo. A queste posizioni Eco oppone l'idea che non si possano scambiare le interpretazioni (o le misinterpretazioni) di due testi differenti: un'interpretazione valida per qualsiasi messaggio non sarebbe, infatti, l'interpretazione di alcun messaggio, ma un testo autonomo che esisterebbe di per se stesso senza l'esigenza di un altro testo come proprio parametro.

Allo schematismo dell'opposizione tra un approccio generativo ed un approccio interpretativo all'opera—cioè al semplicismo di una polarizzazione della situazione attuale della critica nel contrasto tra indirizzi metodologici che concepiscono l'indagine critica come ricostruzione dell'atto creativo da cui l'opera scaturisce, ed indirizzi teorici che privilegiano la funzione attiva di costruzione, o decostruzione, del testo svolta dall'atto della lettura-Eco preferisce la più sfumata tricotomia tra interpretazione come ricerca dell'intentio aucioris, dell'intentio operis, dell'intentio lectoris. Se è vero che l'opera può legittimamente essere concepita come il documento di una intenzione operativa dell'autore, è però anche vero che tale intenzione non si realizza quasi mai pienamente nell'opera, rimanendo, per di più, inattingibile all'interprete. Da tale consapevolezza non discende però conseguentemente, come taluni pretenderebbero, la necessità di sbilanciare l'attenzione ermeneutica esclusivamente sul polo opposto dell'intentio lectoris. Tra i due estremi si pone il testo nella sua evidenza di struttura comunicativa dotata in primo luogo di un senso letterale, che rappresenta, secondo Eco, l'esclusivo punto da cui possono prendere inizio le più disparate avventure esegetiche. Ogni discorso sulla libertà dell'interpretazione non può, infatti, che cominciare da una difesa del senso letterale, "che è quello elencato al primo posto dai dizionari, ovvero quello che ogni uomo della strada definirebbe per primo quando gli venga chiesto cosa significa una data parola" (Limiti 9). Innanzitutto, dunque, un appello al buon senso di chi professandosi interprete non può che intendere il rispetto del testo, anziché come un obbligo improbabile nei confronti dell'autore e dell'opera, come un dovere nei confronti della propria stessa intelligenza. Solo dopo questa iniziale restrizione sarà allora possibile distinguere tra un'interpretazione semantica (o semiosica) ed un'interpretazione critica (o semiotica), cioè tra il risultato del processo per cui il destinatario, posto di fronte alla manifestazione lineare del testo lo riempie di senso e l'operazione mediante la quale si cerca di spiegare perché un testo possa produrre quelle (o altre alternative) interpretazioni. Due aspetti, questi, che sono strettamente vincolati l'uno all'altro, tra i quali pero Eco sembra stabilire una gerarchia di priorità che attribuisce la precedenza operativa all'individuazione dei 
pattern formali costituenti la struttura significativa dell'opera, in modo tale da fissare i punti di riferimento necessari ad un'appropriato recupero dei significati.

L'interprete viene quindi chiamato ad una collaborazione responsabile, e cioè a riempire i vuoti semantici dell'opera e a scegliere i propri percorsi di lettura, verificando però ogni volta le proprie presupposizioni nel corpo del testo. Leggere significa innanzitutto formulare delle ipotesi, individuare la fisionomia di quel Lettore Modello che ogni strategia di scrittura presuppone, e quindi stabilire con un'operazione abduttiva una relazione coerente fra dati testuali differenti. Cio che però distingue l'interpretazione dal semplice uso ${ }^{7}$ dei testi è la necessità che le ipotesi ermeneutiche suggerite da determinati elementi dell'opcra vengano confermate dal suo insieme. Usare un testo significa beneficiare degli stimoli che da esso si possono trarre per muovere verso direzioni che conducono al di fuori dell'universo di discorso che il testo apre; interpretarlo, invece, vuol dire rintracciare, seppure servendosi di strumenti analitici personali, le relazioni e le implicazioni che si danno all'interno di quell'universo. Si possono formulare infinite ipotesi interpretative, ma il confronto con la coerenza del testo non potrà che imporre il ripudio di congetture che non trovino fondamento nell'opera considerata nel suo insieme. Il testo può infatti essere inteso come un sistema di relazioni interne che consente l'attuazione di certi collegamenti possibili, ma allo stesso tempo ne impedisce altri. "Prima che un testo venga prodotto, potrebbe essere inventata ogni sorta di testo. Dopo che un testo è stato prodotto, è possibile fargli dire molte cose-in certi casi un numero potenzialmente infinito di cose - ma è impossibile —o almeno criticamente illegittimo-fargli dire ciò che non dice" (Limiti 107).

Si tratta, insomma, di verificare se le energie ermeneutiche spese dall'interprete siano confortate dalle risorse dell'oggetto che indaga. A questo proposito, Eco nota come gli eccessi interpretativi a cui si abbandonano alcuni critici contemporanei siano caratterizzabili proprio nei termini di una trasgressione ad elementari criteri di economia dell'interpretazione. A fondamento di questo immotivato dispendio di energie esegetiche, Eco individua la disposizione ad una lettura sospettosa del testo che, ancora una volta, riconduce ad un denominatore comune le recenti teorie dell'interpretazione e l'atteggiamento ermetico. Tale disposizione nei confronti del testo si manifesta come una propensione ad un eccesso di meraviglia, cioè come un'inclinazione ossessiva a ritenerne gli elementi più superficiali come indizi di un significato nascosto, mentre proprio la loro evidenza dovrebbe indurre a spiegarli in termini molto più economici. È chiaro che la curiosità ed il sospetto non debbano essere considerati di per sé patologici, ma alla condizione che si assuma un determinato segno come indizio solo se sono soddisfatte tre condizioni: "che non possa essere spiegato in modo più economico, che punti verso una sola causa (o a una ristretta classe di cause possibili) e non a una pluralità indeterminata e difforme di cause, e che possa far sistema con altri indizi" (Limiti 86).

La proposta di Eco è quindi quella di un salutare utilitarismo ermeneutico, 
che imponga, nel valutare una congettura interpretativa, di prendere attentamente in considerazione il rapporto tra i costi in termini di energia ermeneutica spesa, ed $\mathrm{i}$ benefici che se ne ottengono in termini di comprensione dell'opera. Il principio che si fa garante della possibilità di un bilancio positivo di questo rapporto è, per Eco, il consenso di "una comunità di interpreti intesa a raggiungere un qualche accordo, se non sulle interpretazioni migliori, almeno sul rifiuto di quelle insostenibili" (Limiti 11). Piuttosto che appellarsi ad un'improbabile verità assoluta del testo o arrendersi ad un relativismo pessimistico, che consideri impossibile ogni presa conoscitiva sulla realtà, l'interprete può ricorrere ad un concetto regolativo di significato che, se non è oggettivo, è almeno intersoggettivo ed è quindi privilegiato rispetto a qualsiasi significato che non riscuota il consenso della comunità. ${ }^{8}$ Si tratta però di un esito non esente da una certa ambiguità: sembrerebbe infatti che ritenendo accettabile solo ciò su cui la comunità è indotta a concordare, unico giudice dell'appropriatezza di un'interpretazione debba divenire il "successo" che essa può raggiungere in seno alla comunità degli interpreti. Ma davvero quella che ottiene maggiore successo è sempre l'ipotesi più ragionevole? (se così fosse, vista la popolarità che ha raggiunto in America, il decostruzionismo non potrebbe che essere considerato come l'approccio più adeguato). È vero che Eco parla della comunità degli interpreti nei termini di un'istanza trascendentale, svincolandone così il concetto dai condizionamenti contingenti indotti da un qualsiasi paradigma, ${ }^{9}$ ma si potrebbe obiettare che operativamente questa sorta di Tribunale Supremo della Ragione è necessariamente costituito da chiunque pensa con i mezzi che la sua condizione di uomo collocato nel mondo e nella storia gli formiscono. Non si rischia allora di scadere in un appiattimento della ricerca sui criteri riconosciuti all'interno del sistema culturale esistente, con il pericoloso risultato di mettere la museruola alla fantasia e all'invenzione di nuove prospettive? La storia della scienza mostra chiaramente come il progresso scientifico molto spesso scaturisca dall'invenzione di nuove regole che trasgrediscono apertamente quelle stabilite all'interno di una dato orizzonte conoscitivo. È proprio ciò che destabilizza il sistema delle conoscenze, e che quindi può apparire in un primo tempo come un semplice paralogismo, a promuovere quelle innovazioni che il sistema non prevedeva. Come ha scritto Lyotard, in rapporto all'ideale di trasparenza sotteso al criterio consensuale di legittimazione del sapere, questa proprietà destabilizzante della conoscenza "costituisce un fattore di opacità, che rinvia il momento del consenso" (111-12) e pone piuttosto l'accento su quello del dissenso. È chiaro che quando il dissenso diventi una pratica fine a se stessa, uno strumento artificioso volto solo alla ricerca dell'effetto, esso perde tutta la sua carica trasgressiva ed il suo potenziale conoscitivo; è però importante salvaguardare le possibilità di un dissenso costruttivo che, resistendo a tentazioni nichilistiche, si adoperi positivamente per una evoluzione del sapere.

Un'analoga ambiguità sembra caratterizzare il discorso di Eco quando afferma che nel processo di semiosi illimitata "è possibile andare da qualsiasi nodo a 
ogni altro nodo, ma i passaggi sono regolati da regole di connessione che la loro storia culturale ha in qualche modo legittimato" (Limiti 107). Anche in questo caso, il vincolo che limita gli itinerari interpretativi possibili solo a quelli che hanno ricevuto il crisma di una tradizione culturale consolidata sembrerebbe impedire ogni svolta innovativa, e condannare l'attività di ricerca a rovistare nel bagaglio enciclopedico acquisito. In realtà la posizione di Eco si fonda su di una concezione ben più articolata, e che, per essere compresa nella sua effettiva portata, impone di ripercorrere, almeno sinteticamente, l'elaborazione della categoria di semiosi illimitata. Ispirandosi alla semiologia di Peirce, Eco ha posto a fondamento del proprio sistema semiologico l'idea che il referente di un segno non sia altro che un'unità culturale. Ciò significa che ogni volta che si cerca di determinare il significato di un segno non si può far altro che riproporlo nei termini di un'entità astratta che è, a sua volta, un segno. Ogni significante rimanda quindi ad un altro significante che ne spieghi il significato, ma che, in quanto significante, ha bisogno di un altro significante che lo spieghi, e così via. Ognuno di questi significanti che spiegano i significati di significanti precedenti rappresenta quello che Peirce ha chiamato l'interpretante di un segno, e la successione degli interpretanti è stata definita appunto da Eco come un processo di semiosi illimitata. Nel Trattato di semiotica generale, la fertilità di questa categoria gli sembrava consistere nel mostrarci come la significazione e la comunicazione, per mezzo di spostamenti continui che riferiscono un segno ad altri segni, "circoscrivano le unità culturali in modo asintotico, senza mai arrivare a 'toccarle' direttamente ma rendendole di fatto accessibili attraverso altre unità culturali" (104). La somma dei vari linguaggi veniva così presentata come un sistema autoesplicativo che si spiega per successivi sistemi di convenzione che si chiariscono l'un l'altro (100). Proprio questa autonomia del sistema semiotico si prestava però ad essere interpretata come un ripiegamento del sistema su se stesso, cioè come l'incapacità dell'universo semiotico, che si compie nell'intrecciarsi ludico di rimandi infiniti da un segno all'altro, a trovare alcun punto di contatto con l'universo extrasemiotico. Riconsiderando, in Lector in fabula, la teoria peirciana dell'interpretante Eco perviene ad una ulteriore messa a punto della sua rielaborazione delle posizioni di Peirce. Se ogni segno interpreta un altro segno "e la condizione basilare della semiosi è proprio questa condizione di regresso infinito" (Lector 38), ciò non significa che il succedersi dei rimandi renda inattingibile quell'Oggetto Dinamico che, secondo Peirce, rappresenta la finalità extrasemiotica che determina ogni atto semiosico. Producendo una serie di risposte immediate (che sono i suoi Interpretanti Energetici) un segno "stabilisce a poco a poco una abitudine (habit), una regolarità di comportamento nel proprio interprete" (Lector 43). L'intervenire di una abitudine, che modifica permanentemente o transitoriamente il nostro modo di agire nel mondo, rappresenta quello che Peirce chiama l'Interpretante Finale, raggiunto il quale "la semiosi illimitata si arresta, lo scambio dei segni ha prodotto modificazioni dell'esperienza, l'anello mancante tra semiosi e realtà fisica è stato finalmente identi- 
ficato" (Lector 45). Il significato si produce quindi attraverso l'istituzione di un'abitudine che divenendo patrimonio comune di una determinata comunità di parlanti un dato linguaggio, fonda la possibilità della comunicazione tra i membri della comunità stessa. Nessuno infatti può usare un linguaggio se non rispondendo idealmente dell'osservanza delle regole di quel linguaggio davanti alla comunità dei parlanti, e queste regole, che non sono altro che abitudini istituite come leggi, sono legittimate dalla loro ricorrenza nella storia culturale della comunità. Dire quindi che nell'interpretare un segno lo si possa riferire solo ad una stratificazione di sensi, le cui connessioni con il segno da interpretare siano autorizzate da una tradizione, non significa altro che ribadire la necessità che la comunicazione si fondi su dati comuni (le abitudini appunto) al mittente ed al destinatario di un messaggio, in quanto membri di una determinata comunità linguistica. Le possibilità conoscitive della semiosi non si esauriscono però nel raggiungimento dell'Interpretante Finale, che non deve essere inteso come tale in senso cronologico. "La semiosi muore in ogni momento, ma come muore risorge dalla proprie ceneri. ... Il sistema dei sistemi semiotici, che potrebbe sembrare un universo culturale idealisticamente separato dalla realtà, di fatto porta ad agire sul mondo e a modificarlo; ma ciascuna azione modificatrice si converte a propria volta in segno e dà origine a un nuovo processo semiosico" (Lector 46). La forza dei segni è quella di tradursi pragmaticamente in azioni che modificano il mondo, la loro ricchezza consiste, invece, nel fatto che divenendo azioni essi si trasformano in nuovi segni, dando inizio a nuovi processi di interpretazione, dai quali possono scaturire nuovi ed imprevedibili orizzonti.

Sulla base di questa concezione, Eco contesta (Limiti 330-38) la lettura che Derrida fa di Peirce, nel secondo capitolo di De la grammatologie. Il filosofo francese si rifà infatti alla nozione di semiosi illimitata per legittimare il suo tentativo di delineare una semiosi del gioco infinito, considerando Peirce come un antesignano della decostruzione dell'idea logocentrica di un significato trascendentale, che porrebbe un termine rassicurante al rinvio da segno a segno. Secondo Eco, nonostante le apparenti consonanze, non è possibile assimilare la deriva decostruzionista al concetto di semiosi illimitata. Per Peirce, infatti, l'idea di significato è tale da implicare qualche riferimento ad uno scopo, cioè ad un fine extrasemiotico dell'interpretazione. Questo fine extrasemiotico è quello che Peirce chiama l'Oggetto Dinamico, e cioè quello stato del mondo esterno che determina la produzione del segno. L'attività interpretativa non può quindi essere intesa come ludico gioco combinatorio di associazioni e rimandi, ma è un'operazione volta a promuovere un'approssimazione asintotica all'Oggetto Dinamico, mediante una sempre maggiore determinazione dei segni sia per quanto riguarda l'estensione che l'intenzione. Anche quando un rappresentamen complesso, come nel caso di un testo, acquisisca un'indipendenza dall'intenzione del suo enunciatore e si presenti al fruitore nella sua autonomia, esso diviene, per la sua stessa evidenza di Oggetto Testuale, "l'Oggetto Dinamico rispetto al quale ogni interpretazione fornisce l'Oggetto Immediato corrispondente" (Limiti 335). 
Se quindi è vero che ogni segno si presenta come un coacervo di possibili sviluppi testuali, nel momento in cui esso viene a trovarsi collocato all'interno di un testo, la ricchezza enciclopedica dei suoi sensi virtuali è limitata dall'universo di discorso nel quale è inserito. L'interpretazione trova quindi un limite nella natura stessa del proprio oggetto: quando i segni vengono organizzati in un testo si instaurano tra di essi dei rapporti che ne controllano le possibili espansioni significative, sottomettendoli a leggi istituite dalle abitudini comunicative che sono patrimonio collettivo di una determinata comunità di interpreti. Alle nuove teorie letterarie che, considerando ogni interpretazione come una misinterpretazione, sembrano voler abolire qualsiasi criterio valutativo degli enunciati esegetici, Eco obietta dunque che, se è impossibile dire quale sia la migliore delle interpretazioni di un testo, è però almeno possibile-sulla base delle regole, o abitudini, che costituiscono il fondamento della comunicazione all'interno della comunità degli interpreti-stabilire quali ne siano le interpretazioni inaccettabili. In questo senso appellarsi ad un criterio consensuale non significa inibire le possibilità di produrre interpretazioni innovative (possibilità che sono garantite dall'incessante rigenerarsi della semiosi), ma richiamarsi alla nozione di una competenza enciclopedica comune in base al quale gli appartenenti ad una determinata comunità linguistica possono scambiarsi messaggi e concordare sui loro significati. ${ }^{10}$

Mosso dalla particolare congiuntura del dibattito critico attuale, Eco si assume dunque, in questa sua recente opera teorica, l'onere di perorare la causa dell'interpretazione e della sua dipendenza dalla struttura significativa dell'opera. Questa sua presa di posizione non implica però un rinnegamento del rilievo di quella cooperazione interpretativa da parte del lettore, che lui stesso aveva, tra i primi, analizzato in suoi precedenti studi. Insistendo particolarmente sulla natura congetturale dell'interpretazione egli si preoccupa piuttosto di sottolineare, ancora una volta, come l'intenzione del testo e quello del lettore siano strettamente collegate in ogni processo di fruizione. La stessa opposizione tra interpretazione ed uso dei testi gli sembra dover essere considerata non altrimenti che un'astrazione, visto che ogni lettura risulta sempre da un vario articolarsi di questi due atteggiamenti. L'obiettivo del suo discorso non è quindi tanto quello di difendere l'interpretazione per condannare l'uso, quanto quello di smascherare la malafede di quelle letture critiche che abusano del testo imponendogli dei significati arbitrari, di cui giustificano l'evocazione con motivazioni inconsistenti. In questo senso, la proposta di Eco sembra aderire ad una concezione dell'attività interpretativa intesa come paziente ricostruzione della legge, o delle leggi, che governano l'organizzazione dei significati di un'opera; operazione che, come scriveva già nella Struttura assente, si concreta "in un saggiare la forma significante per vedere sino a che punto supporti l'immissione di sensi nuovi, grazie a codici di arricchimento; in un ripudio di codici arbitrari che si inseriscano nel corso dell'interpretazione e non sappiano fondersi con gli altri" (81). Ma lo stesso Eco ci ha mostrato nelle sue opere come questo paziente lavoro di restauro, volto al recupero del significato del testo, non sia un fine in se stesso. La comprensione 
dell'opera non si risolve in un compiacimento estetizzante, nel manifestarsi di una emozione rievocata in tranquillità; l'esperienza dell'opera è soprattutto un'esperienza conoscitiva che modifica la nostra maniera di porci in rapporto con le cose, e lo studio di questa esperienza non può prescindere quindi dal considerare il ruolo che essa gioca nel sistema generale della conoscenza. Si interpreta l'opera perché in qualche modo essa ci è utile per comprendere noi stessi, per indagare i modi differenti in cui l'uomo rappresenta, e quindi conosce, l'universo in cui vive. Assieme ad una critica e ad una teoria letterarie che si interroghino su come leggere è allora essenziale mantenere viva una riflessione sul perché continuare a leggere in un mondo che sembra sempre più emarginare la letteratura, ma che al contempo manifesta un disperato bisogno del contributo conoscitivo che la letteratura sinora ha dato e che ancora può dare. Se, come scriveva Italo Calvino presentando le sue Lezioni americane, la letteratura ha un futuro perché ci sono cose che solo la letteratura può dare con i suoi mezzi specifici (1), allora questa peculiarità pone l'ambito letterario sullo stesso piano di altri ambiti conoscitivi, e ne proietta le linee di forza al di fuori dei ristretti confini disciplinari. Indagare i vettori che si irraggiano da questo movimento di proiezione significa necessariamente confrontare la letteratura con altre dimensioni della cultura.

Ma su quali basi metodologiche è possibile operare questo confronto? Per lo strutturalismo il raffronto tra il dominio dell'arte e gli altri orizzonti della conoscenza poteva avvenire sulla base di una serie di operazioni semplificatrici, che consentivano di uniformare realtà differenti da un solo punto di vista, riducendo $i$ fenomeni culturali a modelli di descrizione. L'assunzione di questa prospettiva monologica, per lungo tempo dominante nell'orizzonte degli studi letterari, è però in molti casi degenerata in una sorta di idolatria del testo, che ha circoscritto le possibilità di intervento critico alla sola dimensione descrittiva. La probabilità di ovviare a questa impasse consiste, forse, in un approccio che spinga in profondita ${ }^{11}$ i dati raccolti sulla superficie testuale, alla ricerca di quei corti circuiti che pongano in relazione aspetti differenti del sapere, promuovendo una riflessione sul ruolo specifico che ciascuno di quegli aspetti assolve nel sistema generale della cultura. Un simile orientamento non si propone più di funzionare come un' argomentazione limitata entro i margini di una data disciplina, ma aspira ad elaborare una riconsiderazione dei rapporti che intercorrono tra le diverse modalità del conoscere. Solo rinunciando alle comode certezze di una visione esclusiva, e assumendo piuttosto una pluralità di punti di vista sarà allora possibile ripensare in una chiave nuova e significativa concetti fondamentali come quelli di scrittura, lettura, tradizione.

II Universita di Roma, Tor Vergata 


\section{NOTE}

1 Del dibattito seguito alla pubblicazione di Opera aperta, lo stesso Eco dà un dettagliato resoconto nell'introduzione alla quarta edizione del libro (IX-XXII).

2 Sull'operatività dell'elaborazione di schemi strutturali, o codici, di rappresentazione della configurazione semiotica dei testi, sul loro valore di modelli conoscitivi, di finzioni operative da non confondere con la realtà oggettiva di ciò che sono preposti a descrivere, Eco insiste particolarmente nella Struttura assente (259-65, 354-60).

3 La piena identificazione delle regole che costituiscono l'idioletto estetico risulta però possibile solo per opere caratterizzate da un elevato livello di standardizzazione, tanto che lo stesso Eco ("La critica") ha provocatoriamente proposto che di critica propriamente semiologica si possa parlare solo per opere nelle quali ad ogni livello ricorra una legge estremamente semplice ed evidente.

$4 \mathrm{Nel}$ suo After the New Criticism, lo studioso americano Frank Lentricchia sintetizza in questo elenco i vari orientamenti che coesistono nel panorama della teoria e della critica letteraria americane: critica genealogico-engagé, critica femminista, critica mitografica, critica fenomenologica, critica simbolista, critica formalista, critica strutturalista, critica freudiana, critica esistenzialista, critica marxista, critica sociologica, critica storiografica, critica semiotica, critica ermeneutica, contestualismo neocoleridgiano. A questa lista vanno inoltre aggiunti i residui approcci new critical, il decostruzionismo, il cosiddetto distruzionismo ed il New Historicism.

5 È da notare che in realtà il termine decostruzione è usato solo sporadicamente nell'opera di Jacques Derrida ed in nessuno dei suoi scritti esso viene posto in relazione agli studi letterari: si vedano, in particolare: "Structure", Positions e De la grammatologie.

6 In alcuni casi, come per le opere di Harold Bloom e Geoffrey Hartmann, questa influenza del pensiero ermetico è stata, d'altronde, apertamente riconosciuta.

7 La distinzione fra interpretazione ed uso era stata posta da Eco già in Lector in fabula (59-60).

8 Il riferimento ad una comunità di interpreti accomuna, sebbene con caratterizzazioni molto differenti fra loro, vari indirizzi critici e filosofici: dal reader oriented criticism di Stanley Fish, all'estetica della ricezione, all'ermeneutica di Gadamer. Rispetto a queste prospettive la posizione di Eco è però più vicina a quella di Karl Otto Apel, con la quale condivide il riferimento alla filosofia di Peirce. Nel tentativo di riscattare l'ermeneutica dal relativismo storicista, Apel fa infatti appello ad un criterio regolativo di verità che si esprime "nell' idea della realizzazione d' una comunità illimitata dell' interpretazione che è presupposta implicitamente come istanza generale di controllo da chiunque in generale argomenta (dunque da chiunque pensa!)" (164).

9 Nel senso inteso da Kuhn.

10 Ciò non significa ovviamente che la competenza del destinatario e quella dell'emittente siano necessariamente le stesse, ma che esiste un parametro di confronto intersoggettivo in base al quale valutare le inferenze dei destinatari (Lector 53-56). Eco ha particolarmente insistito sul fatto che la competenza enciclopedica non sia la stessa cosa del codice di jakobsoniana memoria (Trattato 196-200), infatti: "Per 'decodificare' un messaggio verbale occorre, oltre alla competenza linguistica, una competenza variamente circostanziale, una capacità di far scattare presupposizioni, di reprimere idiosincrasie, eccetera eccetera" (I limiti 53).

11 Sul criterio della profondità della comprensione come canone conoscitivo delle scienze umane, contrapposto all'ideale di esattezza della conoscenza nelle scienze naturali si veda Bachtin. 


\section{OPERE CITATE}

Apel, Karl O. "Scientismo o ermeneutica trascendentale?". Comunità e comunicazione. Torino: Rosemberg, 1977. 132-67.

Bachtin, Michail. "Per una metodologia delle scienze umane". L'autore e l'eroe. Teoria letteraria e scienze umane. Torino: Einaudi, 1988. 375-87.

Bloom, Harold. Kabbalah and Criticism. New York: Seabury Press, 1975.

Calvino, Italo. Lezioni americane. Sei proposte per il prossimo millennio. Milano: Garzanti, 1988. Derrida, Jacques. De la grammatologie. Paris: Minuit, 1967.

. Positions. Paris: Minuit, 1972.

"Structure, Sign, and Play in the Discourse of the Human Sciences". The Structuralist Controversy: The Languages of Criticism and the Sciences of Man. Eds. Richard Macksey and Eugenio Donato. Baltimore: Johns Hopkins UP, 1972.

Di Girolamo, Costanzo, Alfonso Berardinelli e Franco Brioschi. La ragione critica. Torino: Einaudi, 1986.

Eco, Umberto. I limiti dell' interpretazione. Milano: Bompiani, 1990.

. "La critica semiologica". I metodi attuali della critica in Italia. Eds. Maria Corti e Cesare Segre. Torino: Eri, 1970. 317-27.

. La struttura assente. La ricerca semiotica e il metodo strutturale. Milano: Bompiani, 1968.

. Lector in fabula. La cooperazione interpretativa nei testi narrativi. Milano: Bompiani, 1979.

. "Le strutture narrative in Fleming". AA.VV. L'analisi del racconto. Milano:

Bompiani, 1969. 123-62.

. Opera aperta. Forma e indeterminazione nelle poetiche contemporanee. Milano:

Bompiani, 1962 [ $4^{\text {a }}$ ediz. riveduta, 1985].

. Trattato di semiotica generale. Milano: Bompiani, 1975.

Hartmann, Geoffrey H. and S. Budick, eds. Midrash and Literature. New Haven: Yale UP, 1986.

Kuhn, Thomas S. The Structure of Scientific Revolutions. Chicago: U of Chicago P, 1962.

Lentricchia, Frank. After the New Criticism. Chicago: U of Chicago P, 1980.

Lyotard, Jean-François. La condizione postmoderna. Milano: Feltrinelli, 1981.

Wimsatt Jr., William K. and Monroe Beardsley. "The Intentional Fallacy". The Verbal Icon. Studies in the Meaning of Poetry. New York: Noonday Press, 1954-66. 